\title{
THE MOSTAR INDEX OF FULLERENES IN TERMS OF AUTOMORPHISM GROUP
}

\author{
Modjtaba Ghorbani* and Shaghayegh Rahmani
}

C 2020 by University of Niš, Serbia | Creative Commons License: CC BY-NC-ND

\begin{abstract}
Let $G$ be a connected graph. For an edge $e=u v \in E(G)$, suppose $n(u)$ and $n(v)$ are respectively, the number of vertices of $G$ lying closer to vertex $u$ than to vertex $v$ and the number of vertices of $G$ lying closer to vertex $v$ than to vertex $u$. The Mostar index is a topological index which is defined as $M o(G)=\sum_{e \in E(G)} f(e)$, where $f(e)=|n(u)-n(v)|$. In this paper, we will compute the Mostar index of a family of fullerene graphs in terms of the automorphism group.
\end{abstract}

Keywords: Automorphism group, Mostar index, group action.

\section{Introduction}

For arbitrary vertices $u$ and $v$ of a graph $G$, the distance $d(u, v)$ is defined as the length of a shortest path connecting $u$ and $v$. For the edge $e=u v \in E(G)$, suppose $n(u)$ and $n(v)$ are respectively, the number of vertices of $G$ lying closer to vertex $u$ than to vertex $v$ and the number of vertices of $G$ lying closer to vertex $v$ than to vertex $u$. The Mostar index is defined as $M o(G)=\sum_{e \in E(G)} f(e)$, where $f(e)=|n(u)-n(v)|$, see [10].

Let $G$ be a group which acts on the non-empty set $\Omega$. The left action $G$ on $\Omega$ induces a group homomorphism $\varphi$ from $G$ into the symmetric group $S_{\Omega}$, that satisfies the following two axioms (where we denote $\varphi(g, \alpha)$ as $\alpha^{g}$ ): $\alpha^{e}=\alpha$ for all $\alpha \in \Omega$ (e denotes the identity element of group $G$ ) and $\alpha^{(g h)}=\left(\alpha^{g}\right)^{h}$ for all $g, h \in G$ and all $\alpha \in \Omega$. The orbit of an element $\alpha \in \Omega$ is denoted by $\alpha^{G}$ and it is defined as the set of all $\alpha^{g}$ 's, where $g \in G$. The size of $\Omega$ is called the degree of this action. The stabilizer of an element $\alpha \in \Omega$ is defined as $G_{\alpha}=\left\{g \in G: \alpha^{g}=\alpha\right\}$. Let $H=G_{\alpha}$, then for $\beta \in \Omega(\alpha \neq \beta), H_{\beta}$ is denoted by $G_{\alpha, \beta}$. On the other hand, the orbit-stabilizer theorem implies that $\left|\alpha^{G}\right| \cdot\left|G_{\alpha}\right|=|G|$, see [9].

A bijection $\sigma$ on the vertex set of graph $G$ is called an automorphism of $G$ if it preserves the edge set. In other words, if $\alpha$ is an automorphism of $G$, then $e=u v$

Received January 29, 2019; accepted July 05, 2019

2010 Mathematics Subject Classification. Primary 05C07; Secondary 05C12, 20D45 
is an edge if and only if $\sigma(e)=\sigma(u) \sigma(v)$ is an edge of $G$. Let $A u t(G)$ be the set of all automorphisms of $G$. Then $\operatorname{Aut}(G)$ under the composition of mappings forms a group. The graph $G$ is called vertex-transitive if its automorphism group has one orbit. This means that for two arbitrary vertices $x, y \in V(G)$, there is an autoorphism $\varphi \in \operatorname{Aut}(G)$ such that $\varphi(x)=y$. We can similarly define an edgetransitive graph.

The aim of this paper is to compute the Mostar index of an infinite family of fullerene graphs. To do this, we shall first compute the automorphism group of the fullerene graph, and afterwards we shall compute all edge-orbits. Finally, we shall determine the contribution of each edge in the formula of Mostar index.

\section{Mostar index of fullerenes}

If $G$ is a vertex-transitive graph, for every edge $e=u v \in E(G)$, we have $n(u)=n(v)$ and thus $\operatorname{Mo}(G)=0$. Here, by relyinh on this and knowing that the action of a group on its orbits is transitive, we will compute the Mostar index of a benzenoid graph by means of orthogonal cuts. Let $F$ be an orthogonal cut. For the arbitrary edge $e \in E(G)$, all vertices in one shore of $F$ are closer to the end-vertex of $e$ belonging to the same shore than to the other one. Hence, all edges of the same orthogonal cut contribute equally to $\operatorname{Mo}(B)$. It is proved in [10] that if $F \subset$ $E(B)$ is an orthogonal cut of a benzenoid graph $B$ of size $p$ and if the shores of $F$ have $n_{1}$ and $n_{2}$ vertices, respectively, then the total contribution of edges from $F$ to $\operatorname{Mo}(B)$ is equal to $p\left|n_{1}-n_{2}\right|$. Hence, we have the following theorem.

Theorem 2.1. Let $B$ be a benzenoid graph on $n$ vertices and let $F_{1}, \ldots, F_{q}$ be all its orthogonal cuts. Let $p_{i}$ denotes the size of $F_{i}$, and $n_{i_{1}}$ and $n_{i_{2}}$ be the number of vertices in its shores. Then

$$
\operatorname{Mo}(B)=\sum_{i=1}^{q} p_{i}\left|n_{i_{1}}-n_{i_{2}}\right| .
$$

Došlić et al. in [10] proved that since the dodecahedron and the Buckminster fullerene are the only two vertex-transitive fullerene graphs, we have $\operatorname{Mo}\left(C_{20}\right)=$ $\operatorname{Mo}\left(C_{60}: I_{h}\right)=0$. They also introduced the following open problem:

Problem [10]. Are there other fullerene graphs $G$ such that $\operatorname{Mo}(G)=0$ ?

Here, we will compute the Mostar index of an infinite family of fullerenes. This is the first attempt to give some new results about the above problem. We conjecture that if $F$ is a fullerene (except dodecahedron and the buckminsterfullerene) then $\operatorname{Mo}(F) \neq 0$. Let $F$ be a fullerene with $\operatorname{Mo}(F)=0$. Then for all edges $e=u v$, we have $n(u)=n(v)$ and thus $F$ is a distance balanced graph. In other words, we conjectured that a fullerene is distance-balance if and only if $F$ is vertex-transitive. 
Theorem 2.2. Let $E_{1}, \cdots, E_{r}$ be the orbits of graph $G$ under the action of $A u t(G)$ on the set $E(G)$. Then

$$
\operatorname{Mo}(G)=\sum_{i=1}^{r} \sum_{e_{i} \in E_{i}}\left|E_{i}\right| \times\left|n\left(u_{i}\right)-n\left(v_{i}\right)\right|
$$

Proof. Let $E_{1}, \ldots, E_{r}$ be the orbits of graph $G$ under the action of $A u t(G)$ on the set of edges. For two edges $e=u v$ and $f=a b$ in the same edge-orbit of $G$, one can prove that $\{n(u), n(v)\}=\{n(a), n(b)\}$. This completes the proof.

Fullerenes are polyhedral molecules made entirely of carbon atoms. The most symmetric fullerene is the famous buckminster fullerene, $C_{60}$, whose discovery in 1985 marked the birth of fullerene chemistry [23]. In 1991, the buckminster fullerene was declared "The Molecule of the Year" by Science magazine, and since then, these new graphs have been attracting attention of various research communities. Many methods of graph theory have been applied to investigate the mathematical models of fullerene molecules called fullerene graphs. M. Ghorbani and A. R. Ashrafi, in a series of papers $[1-8,12-17,21]$, introduced some infinite classes of fullerene graphs. At first, they tried to classify fullerenes with respect to their automorphism group. However, this problem is still open, although Fowler and his co-authors in [11] showed that fullerenes are realizable within 28 point groups. Recently, Ghorbani et al. have computed the automorphism group of some classes of polyhedral graphs, see $[18-20]$.

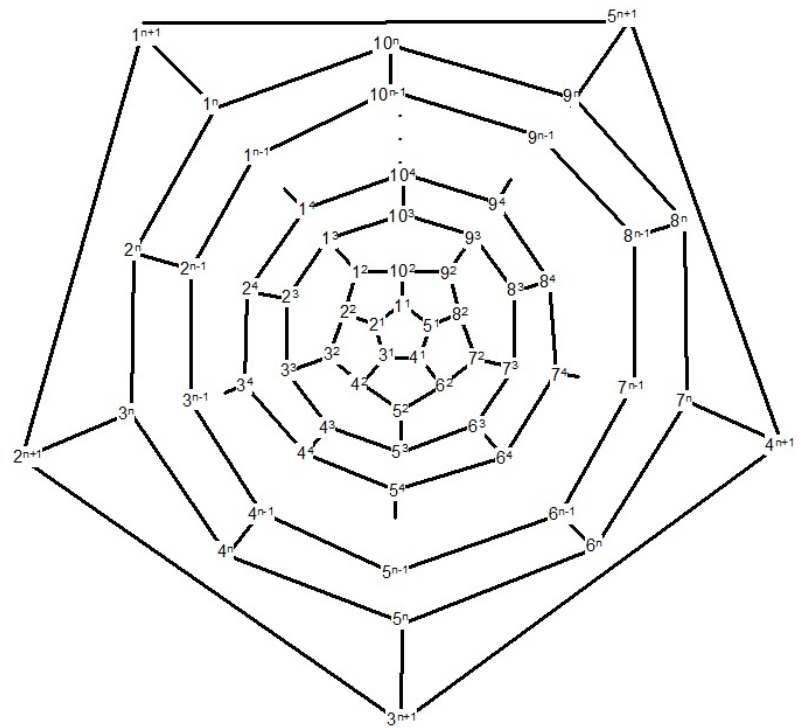

FIG. 2.1: $\mathrm{C}_{10 n}, n$ is even.

Example 2.1. Consider the fullerene graph $C_{10 n}$ ( $n$ is even) as depicted in Figure 2.1. The vertices of central pentagon are labeled by $\left\{1^{1}, 2^{1}, 3^{1}, 4^{1}, 5^{1}\right\}$. These vertices compose 
the first layer of fullerene graph $C_{10 n}$. The vertices of the second layer are the boundary vertices of five pentagons adjacent to the central pentagon and so on. In [22], it is shown that the following elements are in the automorphism group of fullerene graph $C_{10 n}$. Let $\alpha$ be a symmetry element that fixes the vertices $1^{1}, 10^{2}, 10^{3}, \cdots, 10^{n}, 5^{2}, 5^{3}, \cdots, 5^{n}$ and $3^{n+1}$ and $\sigma=\left(1^{1}, 1^{n+1}, 2^{1}, 2^{n+1}, 3^{1}, 3^{n+1}, 4^{1}, 4^{n+1}, 5^{1}, 5^{n+1}\right)\left(1^{2}, 2^{n}, 3^{2}, 4^{n}, 5^{2}, 6^{n}, 7^{2}\right.$, $\left.8^{n}, 9^{2}, 10^{n}\right)\left(2^{2}, 3^{n}, 4^{2}, 5^{n}, 6^{2}, 7^{n}, 8^{2}, 9^{n}, 10^{2}, 1^{n}\right)\left(1^{3}, 2^{n-1}, 3^{3}, 4^{n-1}, 5^{3}, 6^{n-1}, 7^{3}, 8^{n-1}\right.$, $\left.9^{3}, 10^{n-1}\right)\left(2^{3}, 3^{n-1}, 4^{3}, 5^{n-1}, 6^{3}, 7^{n-1}, 8^{3}, 9^{n-1}, 10^{3}, 1^{n-1}\right) \cdots\left(1^{n / 2}, 2^{(n+4) / 2}, 3^{n / 2}\right.$, $\left.4^{(n+4) / 2}, 5^{n / 2}, 6^{(n+4) / 2}, 7^{n / 2}, 8^{(n+4) / 2}, 9^{n / 2}, 10^{(n+4) / 2}\right)\left(2^{n / 2}, 3^{(n+4) / 2}, 4^{n / 2}, 5^{(n+4) / 2}, 6^{n / 2}\right.$, $\left.7^{(n+4) / 2}, 8^{n / 2}, 9^{(n+4) / 2}, 10^{n / 2}, 1^{(n+4) / 2}\right)\left(1^{(n+2) / 2}, 2^{(n+2) / 2}, 3^{(n+2) / 2}, 4^{(n+2) / 2}, 5^{(n+2) / 2}\right.$, $\left.6^{(n+2) / 2}, 7^{(n+2) / 2}, 8^{(n+2) / 2}, 9^{(n+2) / 2}, 10^{(n+2) / 2}\right)$.

It is clear that $\alpha^{2}=\sigma^{10}=1, \alpha \sigma \alpha=\sigma^{-1}$ and $G=\langle\alpha, \sigma\rangle \leqslant A=A u t\left(\mathrm{C}_{10 n}\right)$. On the other hand, every symmetry element which fixes $1^{1}$, must also fix $10^{2}, 10^{3}, \cdots, 10^{n}, 5^{2}, 5^{3}$, $\cdots, 5^{n}$ and $3^{n+1}$. The identity element and the symmetry element $\alpha$ do this, too. Hence, the orbit-stabilizer property ensures that $|A|=\left|1^{1}{ }^{A}\right| \cdot\left|A_{1^{1}}\right|$ and thus $|A|=10 \times 2=20$ which implies that $A \cong D_{20}$. All orbits of the automorphism group $C_{10 n}$ are given in Table 1.

Example 2.2. Consider the fullerene graph $C_{10 n}$ ( $n$ is odd) as depicted in Figure 2.2. Assume that $\alpha$ is a symmetry element which fixes the points $1^{1}, 10^{2}, 10^{3}, \cdots, 10^{n}, 1^{n+1}$, $5^{2}, 5^{3}, \cdots, 5^{n-1}$ and $5^{n}$ and $\sigma$ is a symmetry element by the following permutation presentation:

$\sigma=\left(1^{1}, 4^{n+1}, 2^{1}, 5^{n+1}, 3^{1}, 1^{n+1}, 4^{1}, 2^{n+1}, 5^{1}, 3^{n+1}\right)\left(1^{2}, 7^{n}, 3^{2}, 9^{n}, 5^{2}, 1^{n}, 7^{2}, 3^{n}, 9^{2}, 5^{n}\right)$ $\left(2^{2}, 8^{n}, 4^{2}, 10^{n}, 6^{2}, 2^{n}, 8^{2}, 4^{n}, 10^{2}, 6^{n}\right)\left(1^{3}, 7^{n-1}, 3^{3}, 9^{n-1}, 5^{3}, 1^{n-1}, 7^{3}, 3^{n-1}, 9^{3}, 5^{n-1}\right)\left(2^{3}\right.$, $\left.8^{n-1}, 4^{3}, 10^{n-1}, 6^{3}, 2^{n-1}, 8^{3}, 4^{n-1}, 10^{3}, 6^{n-1}\right) \cdots\left(1^{(n+1) / 2}, 7^{(n+3) / 2}, 3^{(n+1) / 2}, 9^{(n+3) / 2}\right.$, $\left.5^{(n+1) / 2}, 1^{(n+3) / 2}, 7^{(n+1) / 2}, 3^{(n+3) / 2}, 9^{(n+1) / 2}, 5^{(n+3) / 2}\right)\left(2^{(n+1) / 2}, 8^{(n+3) / 2}, 4^{(n+1) / 2}\right.$, $\left.10^{(n+3) / 2}, 6^{(n+1) / 2}, 2^{(n+3) / 2}, 8^{(n+1) / 2}, 4^{(n+3) / 2}, 10^{(n+1) / 2}, 6^{(n+3) / 2}\right)$.

Similar to the last case, one can see $G=\langle\alpha, \sigma\rangle=\operatorname{Aut}\left(\mathrm{C}_{10 n}\right)$ is isomorphic with the dihedral group $D_{20}$. The orbits of the automorphism group are given in Table 4 .

In the following part, we will count all orbits of fullerene $C_{10 n}$. To do this, let fix $(g)$ be the set of elements of $X$ fixed by $g$. By applying Burnside's Lemma, if group $G$ acts on the set $X$, then for $g \in G$, the number of orbits is

$$
\# O=\frac{1}{|G|} \sum_{g \in G}|f i x(g)| .
$$

For every edge $e=u v$ and each automorphism $\alpha \in \operatorname{Aut}(G)$, define $\bar{\alpha}(e)=$ $\{\alpha(u), \alpha(v)\}$. Thus, $\operatorname{Aut}(G)$ acts on the set of edges by the above rule and the Burnside's Lemma for the set of edges can be rewritten as follows:

$$
\# \bar{O}=\frac{1}{|\bar{G}|} \sum_{\bar{g} \in \bar{G}}|f i x(\bar{g})| .
$$

Again, consider the fullerene graph $C_{10 n}$, where $n$ is even, as depicted in Figure 2.1. In this part, we will find the permutation presentation of elements of $A u t\left(C_{10 n}\right)$. 


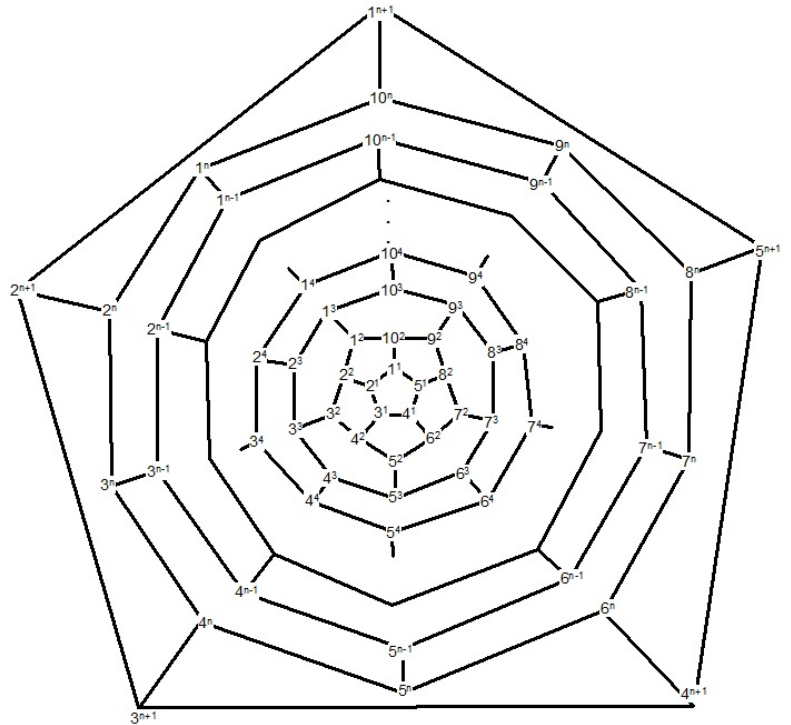

FIG. 2.2: $\mathrm{C}_{10 n}, n$ is odd.

It is not difficult to see that there are five symmetry elements of order two in Aut $\left(C_{10 n}\right)$ denoted by $\alpha_{i}, 1 \leq i \leq 5$. One can easily check that

fix $\left(\alpha_{1}\right)=\left\{1^{1}, 10^{2}, 10^{3}, \cdots, 10^{n}, 5^{2}, 5^{3}, \cdots, 5^{n}, 3^{n+1}\right\}$,

$f i x\left(\alpha_{2}\right)=\left\{2^{1}, 2^{2}, 2^{3}, \cdots, 2^{n}, 7^{2}, 7^{3}, \cdots, 7^{n}, 4^{n+1}\right\}$,

fix $\left(\alpha_{3}\right)=\left\{3^{1}, 4^{2}, 4^{3}, \cdots, 4^{n}, 9^{2}, 9^{3}, \cdots, 9^{n}, 5^{n+1}\right\}$,

fix $\left(\alpha_{4}\right)=\left\{4^{1}, 6^{2}, 6^{3}, \cdots, 6^{n}, 1^{2}, 1^{3}, \cdots, 1^{n}, 1^{n+1}\right\}$,

$f i x\left(\alpha_{5}\right)=\left\{5^{1}, 8^{2}, 8^{3}, \cdots, 8^{n}, 3^{2}, 3^{3}, \cdots, 3^{n}, 2^{n+1}\right\}$.

This means that $\left|f i x\left(\alpha_{i}\right)\right|=2 n,(1 \leqslant i \leqslant 5)$. Suppose $\beta_{1}$ is an involution that maps $1^{1}$ to $2^{n+1}, 2^{1}$ to $1^{n+1}, 3^{1}$ to $5^{n+1}, 4^{1}$ to $4^{n+1}, 5^{1}$ to $3^{n+1}, 1^{2}$ to $2^{n}, 2^{2}$ to $1^{n}$, $3^{2}$ to $10^{n}, 4^{2}$ to $9^{n}, 5^{2}$ to $8^{n}, 6^{2}$ to $7^{n}, 7^{2}$ to $6^{n}, 8^{2}$ to $5^{n}, 9^{2}$ to $5^{n}, 10^{2}$ to $3^{n}$ and so on. It is clear that fix $\beta_{1}=\phi$. If we continue with this method, all permutation presentations of $\beta_{i}$ 's are as follows:

$\beta_{1}=\left(1^{1}, 2^{n+1}\right)\left(2^{1}, 1^{n+1}\right)\left(3^{1}, 5^{n+1}\right)\left(4^{1}, 4^{n+1}\right)\left(5^{1}, 3^{n+1}\right)\left(1^{2}, 2^{n}\right)\left(2^{2}, 1^{n}\right)\left(3^{2}, 10^{n}\right)\left(4^{2}\right.$, $\left.9^{n}\right)\left(5^{2}, 8^{n}\right)\left(6^{2}, 7^{n}\right)\left(7^{2}, 6^{n}\right)\left(8^{2}, 5^{n}\right)\left(9^{2}, 4^{n}\right)\left(10^{2}, 3^{n}\right)\left(1^{3}, 2^{n-1}\right)\left(2^{3}, 1^{n-1}\right)\left(3^{3}, 10^{n-1}\right)$ $\left(4^{3}, 9^{n-1}\right)\left(5^{3}, 8^{n-1}\right)\left(6^{3}, 7^{n-1}\right)\left(7^{3}, 6^{n-1}\right)\left(8^{3}, 5^{n-1}\right)\left(9^{3}, 4^{n-1}\right)\left(10^{3}, 3^{n-1}\right) \cdots\left(1^{n / 2}\right.$, $\left.2^{(n+4) / 2}\right)\left(2^{n / 2}, 1^{(n+4) / 2}\right)\left(3^{n / 2}, 10^{(n+4) / 2}\right)\left(4^{n / 2}, 9^{(n+4) / 2}\right)\left(5^{n / 2}, 8^{(n+4) / 2}\right)\left(6^{n / 2}\right.$, $\left.7^{(n+4) / 2}\right)\left(7^{n / 2}, 6^{(n+4) / 2}\right)\left(8^{n / 2}, 5^{(n+4) / 2}\right)\left(9^{n / 2}, 4^{(n+4) / 2}\right)\left(10^{n / 2}, 3^{(n+4) / 2}\right)\left(1^{(n+2) / 2}\right.$, $\left.2^{(n+2) / 2}\right)\left(3^{(n+2) / 2}, 10^{(n+2) / 2}\right)\left(4^{(n+2) / 2}, 9^{(n+2) / 2}\right)\left(5^{(n+2) / 2}, 8^{(n+2) / 2}\right)\left(6^{(n+2) / 2}\right.$, $\left.7^{(n+2) / 2}\right)$,

$\beta_{2}=\left(1^{1}, 3^{n+1}\right)\left(2^{1}, 2^{n+1}\right)\left(3^{1}, 1^{n+1}\right)\left(4^{1}, 5^{n+1}\right)\left(5^{1}, 4^{n+1}\right)\left(1^{2}, 4^{n}\right)\left(2^{2}, 3^{n}\right)\left(3^{2}, 2^{n}\right)\left(4^{2}\right.$ , $\left.1^{n}\right)\left(5^{2}, 10^{n}\right)\left(6^{2}, 9^{n}\right)\left(7^{2}, 8^{n}\right)\left(8^{2}, 7^{n}\right)\left(9^{2}, 6^{n}\right)\left(10^{2}, 5^{n}\right)\left(1^{3}, 4^{n-1}\right)\left(2^{3}, 3^{n-1}\right)\left(3^{3}, 2^{n-1}\right)$ $\left(4^{3}, 1^{n-1}\right)\left(5^{3}, 10^{n-1}\right)\left(6^{3}, 9^{n-1}\right)\left(7^{3}, 8^{n-1}\right)\left(8^{3}, 7^{n-1}\right)\left(9^{3}, 6^{n-1}\right)\left(10^{3}, 5^{n-1}\right) \cdots\left(1^{n / 2}\right.$, $\left.4^{(n+4) / 2}\right)\left(2^{n / 2}, 3^{(n+4) / 2}\right)\left(3^{n / 2}, 2^{(n+4) / 2}\right)\left(4^{n / 2}, 1^{(n+4) / 2}\right)\left(5^{n / 2}, 10^{(n+4) / 2}\right)\left(6^{n / 2}\right.$, $\left.9^{(n+4) / 2}\right)\left(7^{n / 2}, 8^{(n+4) / 2}\right)\left(8^{n / 2}, 7^{(n+4) / 2}\right)\left(9^{n / 2}, 6^{(n+4) / 2}\right)\left(10^{n / 2}, 5^{(n+4) / 2}\right)\left(1^{(n+2) / 2}\right.$, 


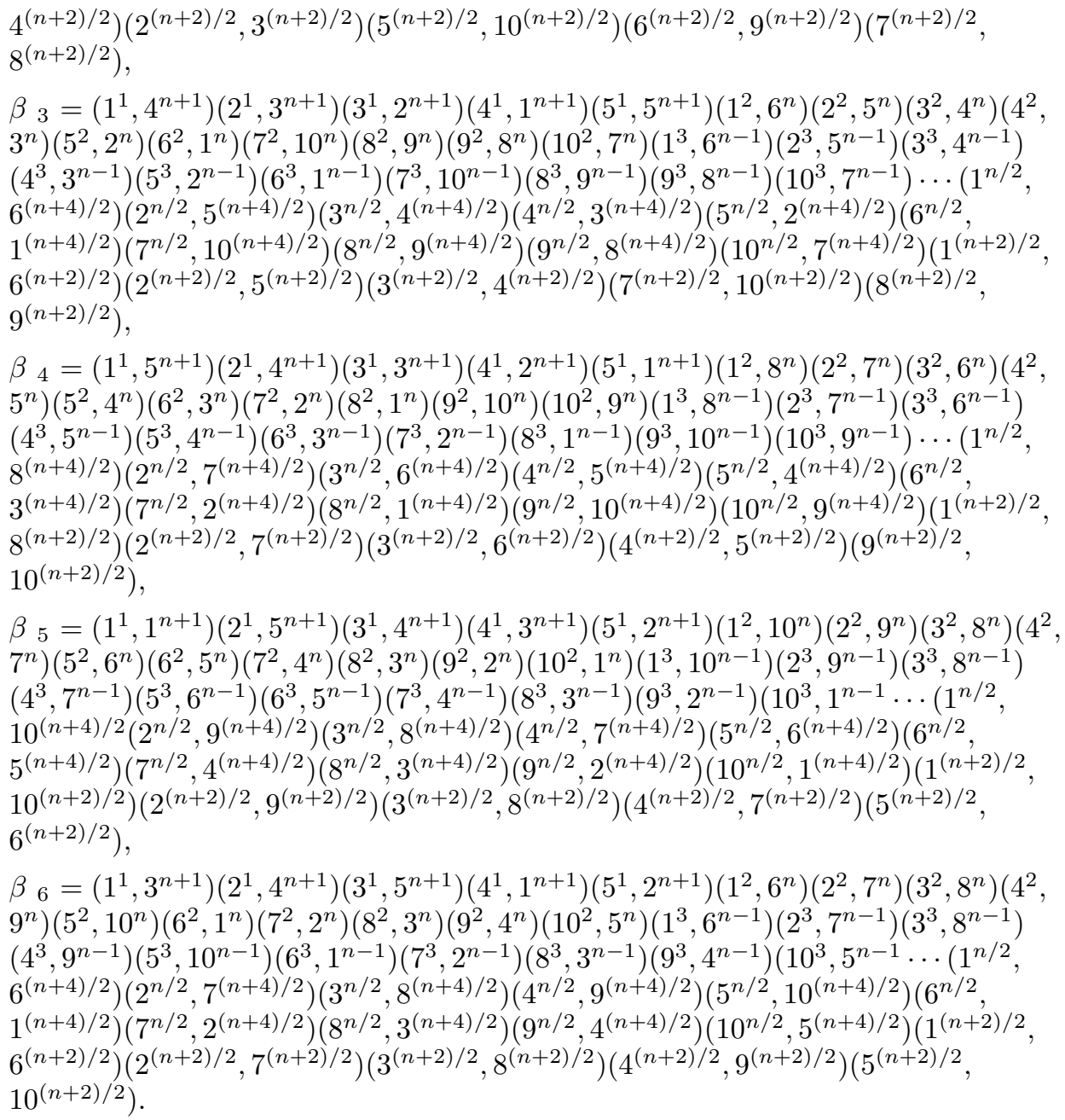

This yields that $A u t\left(C_{10 n}\right)$ includes four rotational elements $\gamma_{i}(1 \leq i \leq 4)$ and four permutations $\sigma_{i}(1 \leq i \leq 4)$ of order 10 with the following permutation presentation:

$\gamma_{1}=\left(1^{1}, 2^{1}, 3^{1}, 4^{1}, 5^{1}\right)\left(1^{2}, 3^{2}, 5^{2}, 7^{2}, 9^{2}\right)\left(2^{2}, 4^{2}, 6^{2}, 8^{2}, 10^{2}\right)\left(1^{3}, 3^{3}, 5^{3}, 7^{3}, 9^{3}\right)\left(2^{3}, 4^{3}\right.$, $\left.6^{3}, 8^{3}, 10^{3}\right) \cdots\left(1^{n-1}, 3^{n-1}, 5^{n-1}, 7^{n-1}, 9^{n-1}\right)\left(2^{n-1}, 4^{n-1}, 6^{n-1}, 8^{n-1}, 10^{n-1}\right)\left(1^{n}, 3^{n}\right.$, $\left.5^{n}, 7^{n}, 9^{n}\right)\left(2^{n}, 4^{n}, 6^{n}, 8^{n}, 10^{n}\right)\left(1^{n+1}, 2^{n+1}, 3^{n+1}, 4^{n+1}, 5^{n+1}\right)$,

$\gamma_{2}=\left(1^{1}, 3^{1}, 5^{1}, 2^{1}, 4^{1}\right)\left(1^{2}, 5^{2}, 9^{2}, 3^{2}, 7^{2}\right)\left(2^{2}, 6^{2}, 10^{2}, 4^{2}, 8^{2}\right)\left(1^{3}, 5^{3}, 9^{3}, 3^{3}, 7^{3}\right)\left(2^{3}, 6^{3}\right.$, $\left.10^{3}, 4^{3}, 8^{3}\right) \cdots\left(1^{n-1}, 5^{n-1}, 9^{n-1}, 3^{n-1}, 7^{n-1}\right)\left(2^{n-1}, 6^{n-1}, 10^{n-1}, 4^{n-1}, 8^{n-1}\right)\left(1^{n}, 5^{n}\right.$, $\left.9^{n}, 3^{n}, 7^{n}\right)\left(2^{n}, 6^{n}, 10^{n}, 4^{n}, 8^{n}\right)\left(1^{n+1}, 3^{n+1}, 5^{n+1}, 2^{n+1}, 4^{n+1}\right)$,

$\gamma_{3}=\left(1^{1}, 5^{1}, 4^{1}, 3^{1}, 2^{1}\right)\left(1^{2}, 9^{2}, 7^{2}, 5^{2}, 3^{2}\right)\left(2^{2}, 10^{2}, 8^{2}, 6^{2}, 4^{2}\right)\left(1^{3}, 9^{3}, 7^{3}, 5^{3}, 3^{3}\right)\left(2^{3}, 10^{3}\right.$, $\left.8^{3}, 6^{3}, 4^{3}\right) \cdots\left(1^{n-1}, 9^{n-1}, 7^{n-1}, 5^{n-1}, 3^{n-1}\right)\left(2^{n-1}, 10^{n-1}, 8^{n-1}, 6^{n-1}, 4^{n-1}\right)\left(1^{n}, 9^{n}\right.$, $\left.7^{n}, 5^{n}, 3^{n}\right)\left(2^{n}, 10^{n}, 8^{n}, 6^{n}, 4^{n}\right)\left(1^{n+1}, 5^{n+1}, 4^{n+1}, 3^{n+1}, 2^{n+1}\right)$, 
$\gamma_{4}=\left(1^{1}, 4^{1}, 2^{1}, 5^{1}, 3^{1}\right)\left(1^{2}, 7^{2}, 3^{2}, 9^{2}, 5^{2}\right)\left(2^{2}, 8^{2}, 4^{2}, 10^{2}, 6^{2}\right)\left(1^{3}, 7^{3}, 3^{3}, 9^{3}, 5^{3}\right)\left(2^{3}, 8^{3}\right.$, $\left.4^{3}, 10^{3}, 6^{3}\right) \cdots\left(1^{n-1}, 7^{n-1}, 3^{n-1}, 9^{n-1}, 5^{n-1}\right)\left(2^{n-1}, 8^{n-1}, 4^{n-1}, 10^{n-1}, 6^{n-1}\right)\left(1^{n}, 7^{n}\right.$, $\left.3^{n}, 9^{n}, 5^{n}\right)\left(2^{n}, 8^{n}, 4^{n}, 10^{n}, 6^{n}\right)\left(1^{n+1}, 4^{n+1}, 2^{n+1}, 5^{n+1}, 3^{n+1}\right)$.

$\sigma_{1}=\left(1^{1}, 5^{n+1}, 5^{1}, 4^{n+1}, 4^{1}, 3^{n+1}, 3^{1}, 2^{n+1}, 2^{1}, 1^{n+1}\right)\left(1^{2}, 10^{n}, 9^{2}, 8^{n}, 7^{2}, 6^{n}, 5^{2}, 4^{n}, 3^{2}\right.$, $\left.2^{n}\right)\left(2^{2}, 1^{n}, 10^{2}, 9^{n}, 8^{2}, 7^{n}, 6^{2}, 5^{n}, 4^{2}, 3^{n}\right)\left(1^{3}, 10^{n-1}, 9^{3}, 8^{n-1}, 7^{3}, 6^{n-1}, 5^{3}, 4^{n-1}, 3^{3}\right.$, $\left.2^{n-1}\right)\left(2^{3}, 1^{n-1}, 10^{3}, 9^{n-1}, 8^{3}, 7^{n-1}, 6^{3}, 5^{n-1}, 4^{3}, 3^{n-1}\right) \cdots\left(1^{n / 2}, 10^{(n+4) / 2}, 9^{n / 2}\right.$, $\left.8^{(n+4) / 2}, 7^{n / 2}, 6^{(n+4) / 2}, 5^{n / 2}, 4^{(n+4) / 2}, 3^{n / 2}, 2^{(n+4) / 2}\right)\left(2^{n / 2}, 1^{(n+4) / 2}, 10^{n / 2}, 9^{(n+4) / 2}\right.$, $\left.8^{n / 2}, 7^{(n+4) / 2}, 6^{n / 2}, 5^{(n+4) / 2}, 4^{n / 2}, 3^{(n+4) / 2}\right)\left(1^{(n+2) / 2}, 10^{(n+2) / 2}, 9^{(n+2) / 2}, 8^{(n+2) / 2}\right.$, $\left.7^{(n+2) / 2}, 6^{(n+2) / 2}, 5^{(n+2) / 2}, 4^{(n+2) / 2}, 3^{(n+2) / 2}, 2^{(n+2) / 2}\right)$,

$\sigma_{2}=\left(1^{1}, 1^{n+1}, 2^{1}, 2^{n+1}, 3^{1}, 3^{n+1}, 4^{1}, 4^{n+1}, 5^{1}, 5^{n+1}\right)\left(1^{2}, 2^{n}, 3^{2}, 4^{n}, 5^{2}, 6^{n}, 7^{2}, 8^{n}, 9^{2}\right.$, $\left.10^{n}\right)\left(2^{2}, 3^{n}, 4^{2}, 5^{n}, 6^{2}, 7^{n}, 8^{2}, 9^{n}, 10^{2}, 1^{n}\right)\left(1^{3}, 2^{n-1}, 3^{3}, 4^{n-1}, 5^{3}, 6^{n-1}, 7^{3}, 8^{n-1}, 9^{3}\right.$, $\left.10^{n-1}\right)\left(2^{3}, 3^{n-1}, 4^{3}, 5^{n-1}, 6^{3}, 7^{n-1}, 8^{3}, 9^{n-1}, 10^{3}, 1^{n-1} \cdots\left(1^{n / 2}, 2^{(n+4) / 2}, 3^{n / 2}\right.\right.$, $\left.4^{(n+4) / 2}, 5^{n / 2}, 6^{(n+4) / 2}, 7^{n / 2}, 8^{(n+4) / 2}, 9^{n / 2}, 10^{(n+4) / 2}\right)\left(2^{n / 2}, 3^{(n+4) / 2}, 4^{n / 2}, 5^{(n+4) / 2}\right.$, $\left.6^{n / 2}, 7^{(n+4) / 2}, 8^{n / 2}, 9^{(n+4) / 2}, 10^{n / 2}, 1^{(n+4) / 2}\right)\left(1^{(n+2) / 2}, 2^{(n+2) / 2}, 3^{(n+2) / 2}, 4^{(n+2) / 2}\right.$, $\left.5^{(n+2) / 2}, 6^{(n+2) / 2}, 7^{(n+2) / 2}, 8^{(n+2) / 2}, 9^{(n+2) / 2}, 10^{(n+2) / 2}\right)$, $\sigma_{3}=\left(1^{1}, 2^{n+1}, 4^{1}, 5^{n+1}, 2^{1}, 3^{n+1}, 5^{1}, 1^{n+1}, 3^{1}, 4^{n+1}\right)\left(1^{2}, 4^{n}, 7^{2}, 10^{n}, 3^{2}, 6^{n}, 9^{2}, 2^{n}\right.$, $\left.5^{2}, 8^{n}\right)\left(2^{2}, 5^{n}, 8^{2}, 1^{n}, 4^{2}, 7^{n}, 10^{2}, 3^{n}, 6^{2}, 9^{n}\right)\left(1^{3}, 4^{n-1}, 7^{3}, 10^{n-1}, 3^{3}, 6^{n-1}, 9^{3}, 2^{n-1}\right.$, $\left.5^{3}, 8^{n-1}\right)\left(2^{3}, 5^{n-1}, 8^{3}, 1^{n-1}, 4^{3}, 7^{n-1}, 10^{3}, 3^{n-1}, 6^{3}, 9^{n-1}\right) \cdots\left(1^{n / 2}, 4^{(n+4) / 2}, 7^{n / 2}\right.$, $\left.10^{(n+4) / 2}, 3^{n / 2}, 6^{(n+4) / 2}, 9^{n / 2}, 2^{(n+4) / 2}, 5^{n / 2}, 8^{(n+4) / 2}\right)\left(2^{n / 2}, 5^{(n+4) / 2}, 8^{n / 2}\right.$, $\left.1^{(n+4) / 2}, 4^{n / 2}, 7^{(n+4) / 2}, 10^{n / 2}, 3^{(n+4) / 2}, 6^{n / 2}, 9^{(n+4) / 2}\right)\left(1^{(n+2) / 2}, 4^{(n+2) / 2}, 7^{(n+2) / 2}\right.$, $\left.10^{(n+2) / 2}, 3^{(n+2) / 2}, 6^{(n+2) / 2}, 9^{(n+2) / 2}, 2^{(n+2) / 2}, 5^{(n+2) / 2}, 8^{(n+2) / 2}\right)$,

$\sigma_{4}=\left(1^{1}, 4^{n+1}, 3^{1}, 1^{n+1}, 5^{1}, 3^{n+1}, 2^{1}, 5^{n+1}, 4^{1}, 2^{n+1}\right)\left(1^{2}, 8^{n}, 5^{2}, 2^{n}, 9^{2}, 6^{n}, 3^{2}, 10^{n}, 7^{2}\right.$, $\left.4^{n}\right)\left(2^{2}, 9^{n}, 6^{2}, 3^{n}, 10^{2}, 7^{n}, 4^{2}, 1^{n}, 8^{2}, 5^{n}\right)\left(1^{3}, 8^{n-1}, 5^{3}, 2^{n-1}, 9^{3}, 6^{n-1}, 3^{3}, 10^{n-1}, 7^{3}\right.$, $\left.4^{n-1}\right)\left(2^{3}, 9^{n-1}, 6^{3}, 3^{n-1}, 10^{3}, 7^{n-1}, 4^{3}, 1^{n-1}, 8^{3}, 5^{n-1}\right) \cdots\left(1^{n / 2}, 8^{(n+4) / 2}, 5^{n / 2}\right.$, $\left.2^{(n+4) / 2}, 9^{n / 2}, 6^{(n+4) / 2}, 3^{n / 2}, 10^{(n+4) / 2}, 7^{n / 2}, 4^{(n+4) / 2}\right)\left(2^{n / 2}, 9^{(n+4) / 2}, 6^{n / 2}, 3^{(n+4) / 2}\right.$, $\left.10^{n / 2}, 7^{(n+4) / 2}, 4^{n / 2}, 1^{(n+4) / 2}, 8^{n / 2}, 5^{(n+4) / 2}\right)\left(1^{(n+2) / 2}, 8^{(n+2) / 2}, 5^{(n+2) / 2}, 2^{(n+2) / 2}\right.$, $\left.9^{(n+2) / 2}, 6^{(n+2) / 2}, 3^{(n+2) / 2}, 10^{(n+2) / 2}, 7^{(n+2) / 2}, 4^{(n+2) / 2}\right)$.

Hence, $\mathrm{C}_{10 n}$ ( $n$ is even) has $\frac{n-2}{2} \times 2+2=n$ orbits each of them has 10 vertices, see Table 1.

\begin{tabular}{cc}
\hline Vertex & Orbit members \\
\hline $1^{1}$ & $1^{1}, 2^{1}, 3^{1}, 4^{1}, 5^{1}, 1^{n+1}, 2^{n+1}, 3^{n+1}, 4^{n+1}, 5^{n+1}$ \\
\hline $1^{2}$ & $1^{2}, 3^{2}, 5^{2}, 7^{2}, 9^{2}, 2^{n}, 4^{n}, 6^{n}, 8^{n}, 10^{n}$ \\
\hline $2^{2}$ & $2^{2}, 4^{2}, 6^{2}, 8^{2}, 10^{2}, 1^{n}, 3^{n}, 5^{n}, 7^{n}, 9^{n}$ \\
\hline$\vdots$ & $\vdots$ \\
\hline $1^{n / 2}$ & $1^{n / 2}, 3^{n / 2}, 5^{n / 2}, 7^{n / 2}, 9^{n / 2}$, \\
& $2^{(n+4) / 2}, 4^{(n+4) / 2}, 6^{(n+4) / 2}, 8^{(n+4) / 2}, 10^{(n+4) / 2}$ \\
\hline $2^{n / 2}$ & $2^{n / 2}, 4^{n / 2}, 6^{n / 2}, 8^{n / 2}, 10^{n / 2}$, \\
& $1^{(n+4) / 2}, 3^{(n+4) / 2}, 5^{(n+4) / 2}, 7^{(n+4) / 2}, 9^{(n+4) / 2}$ \\
\hline $1^{(n+2) / 2}$ & $1^{(n+2) / 2}, 2^{(n+2) / 2}, 3^{(n+2) / 2}, 4^{(n+2) / 2}, 5^{(n+2) / 2}$, \\
& $6^{(n+2) / 2}, 7^{(n+2) / 2}, 8^{(n+2) / 2}, 9^{(n+2) / 2}, 10^{(n+2) / 2}$ \\
\hline
\end{tabular}

Table 1. Members of orbits of $C_{10 n}, n$ is even. 


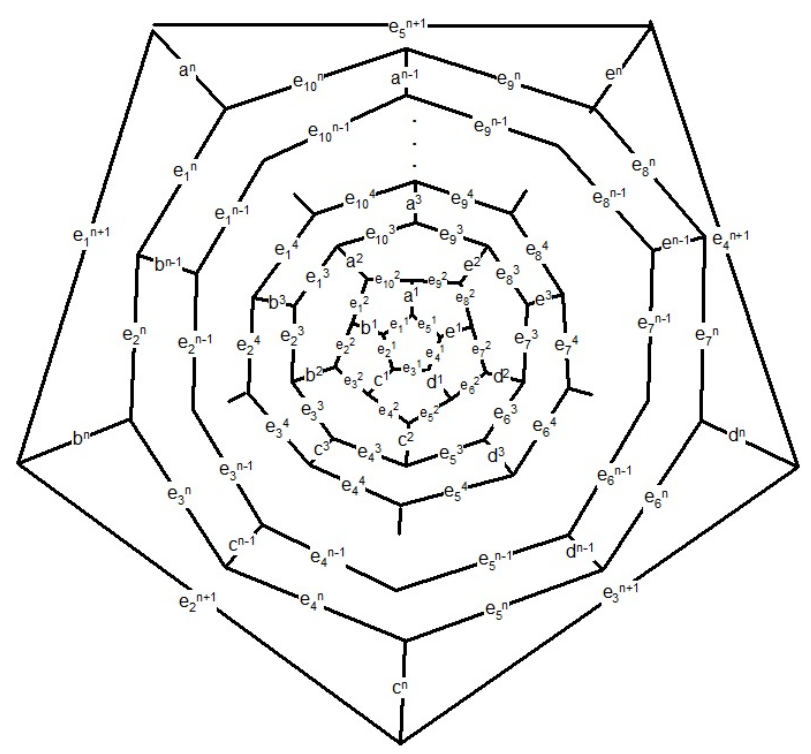

FIG. 2.3: $\mathrm{C}_{10 n}, n$ is even.

It is not difficult to see that $\left|f i x\left(\overline{\alpha_{i}}\right)\right|=n+2(1 \leq i \leq 5),\left|f i x\left(\overline{\beta_{j}}\right)\right|=2$ $(1 \leq j \leq 5)$ and $\mid$ fix $\left(\overline{\beta_{6}}\right)|=|$ fix $\left(\overline{\gamma_{k}}\right)|=|$ fix $\left(\overline{\sigma_{l}}\right) \mid=0(1 \leq k \leq 4),(1 \leq l \leq 4)$. By considering the action of $A u t\left(C_{10 n}\right)$ on the set of edges and using Eq. 2.3, one can prove that the number of orbits is $n+1$ for which $n$ is even. They are $O\left(e_{1}^{1}\right), O\left(e^{1}\right), O\left(e_{1}^{2}\right), O\left(e^{2}\right), \cdots, O\left(e_{1}^{(n-2) / 2}\right), O\left(e^{(n-2) / 2}\right), O\left(e_{1}^{n / 2}\right), O\left(e^{n / 2}\right)$ and $O\left(e_{1}^{(n+2) / 2}\right)$. Hence, we proved the following theorem.

Theorem 2.3. Consider the fullerene graph $C_{10 n}, n$ is even. Then there are $n+1$ orbits under the action of automorphism group on the set of edges.

Theorem 2.4. Consider the fullerene graph $C_{10 n}$, where $n$ is even and $n \geq 10$. Then

$$
M o\left(C_{10 n}\right)=75 n^{2}-100 n+3980 .
$$

Proof. Suppose $e_{1}^{1}=\left\{1^{1}, 2^{1}\right\}$ and $e^{1}=\left\{5^{1}, 8^{2}\right\}$. Then

$$
\begin{aligned}
N_{1^{1}} & =\left\{1^{1}, 5^{1}, 7^{2}, 8^{2}, 9^{2}, 10^{2}, 7^{3}, 8^{3}, 9^{3}, 7^{4}, 8^{4}, 7^{5}\right\}, \\
N_{1^{1}} & =\left\{1^{1}, 5^{1}, 7^{2}, 8^{2}, 9^{2}, 10^{2}, 7^{3}, 8^{3}, 9^{3}, 7^{4}, 8^{4}, 7^{5}\right\}, \\
N_{2^{1}} & =\left\{2^{1}, 3^{1}, 2^{2}, 3^{2}, 4^{2}, 5^{2}, 3^{3}, 4^{3}, 5^{3}, 4^{4}, 5^{4}, 5^{5}\right\}, \\
N_{1^{1}, 2^{1}} & =V\left(C_{10 n}\right)-N_{1^{1}}-N_{2^{1}}, \\
N_{5^{1}} & =\left\{1^{1}, 2^{1}, 3^{1}, 4^{1}, 5^{1}, 2^{2}, 3^{2}, 4^{2}, 3^{3}\right\}, \\
N_{8^{2}} & =V\left(C_{10 n}\right)-N_{1^{1}}-N_{5^{1}}, 8^{2}, \\
N_{5^{1}, 8^{2}} & =\left\{1^{2}, 5^{2}, 6^{2}, 10^{2}, 1^{3}, 2^{3}, 4^{3}, 5^{3}, 2^{4}, 3^{4}, 4^{4}, 3^{5}\right\} .
\end{aligned}
$$


This means that $n\left(1^{1}\right)=12, n\left(2^{1}\right)=12, n\left(1^{1}, 2^{1}\right)=10 n-24, n\left(5^{1}\right)=9, n\left(8^{2}\right)=$ $10 n-21$ and $n\left(5^{1}, 8^{2}\right)=12$. Also if $e_{1}^{2}=\left\{2^{2}, 1^{2}\right\}, e^{2}=\left\{9^{2}, 9^{3}\right\}$. Then

$$
\begin{aligned}
N_{2^{2}} & =\left\{2^{1}, 3^{1}, 4^{1}, 2^{2}, 3^{2}, 4^{2}, 5^{2}, 6^{2}, 3^{3}, 4^{3}, 5^{3}, 6^{3}, 4^{4}, 5^{4}, 6^{4}, 5^{5}, 6^{5}, 6^{6}\right\}, \\
N_{1^{2}} & =V\left(C_{10 n}\right)-N_{2^{2}}-N_{2^{2}, 1^{2}}, \\
N_{2^{2}, 1^{2}} & =\left\{1^{1}, 5^{1}\right\}, \\
N_{9^{2}} & =\left\{1^{1}, 2^{1}, 3^{1}, 4^{1}, 5^{1}, 1^{2}, 2^{2}, 3^{2}, 4^{2}, 5^{2}, 6^{2}, 7^{2}, 8^{2}, 9^{2}, 10^{2}\right\}, \\
N_{9^{3}} & =V\left(C_{10 n}\right)-N_{9^{2}}-N_{9^{2}, 9^{3}}, \\
N_{9^{2}, 9^{3}} & =\varnothing,
\end{aligned}
$$

and thus $n\left(2^{2}\right)=18, n\left(1^{2}\right)=10 n-20, n\left(2^{2}, 1^{2}\right)=2, n\left(9^{2}\right)=15, n\left(9^{3}\right)=10 n-15$, $n\left(9^{2}, 9^{3}\right)=0$, and so on, see Table 2. By using Theorem 2.2, for every edge $e=\{u, v\}$, one can determine the contributions of $n(u)$ and $n(v)$ of edge $e=\{u, v\}$ as reported in Table 2. The summation of these integers yields that

$$
\begin{aligned}
& M o\left(C_{10 n}\right)=10 \times(12-12)+10 \times(10 n-30)+20 \times(10 n-38) \\
& +10 \times(10 n-30)+20 \times(10 n-50)+10 \times(10 n-50) \\
& +20 \times(10 n-65)+10 \times(10 n-70)+20 \times(10 n-81) \\
& +10 \times \sum_{i=0}^{n / 2-5} 10 n-2(45+10 i)+20 \times \sum_{i=0}^{n / 2-6} 10 n-2(50+10 i) \\
& +10 \times(5 n-5 n)=75 n^{2}-100 n+3980 .
\end{aligned}
$$

\begin{tabular}{ccc}
\hline Type of edge & $\boldsymbol{n}(\boldsymbol{u}), \boldsymbol{n}(\boldsymbol{v})$, equidistant & Number \\
\hline$e_{1}^{1}$ & $12,12,10 n-24$ & 10 \\
\hline$e^{1}$ & $9,10 n-21,12$ & 10 \\
\hline$e_{1}^{2}$ & $18,10 n-20,2$ & 20 \\
\hline$e^{2}$ & $15,10 n-15,0$ & 10 \\
\hline$e_{1}^{3}$ & $24,20 n-26,2$ & 20 \\
\hline$e^{3}$ & $25,10 n-25,0$ & 10 \\
\hline$e_{1}^{4}$ & $32,10 n-33,1$ & 20 \\
\hline$e^{4}$ & $35,10 n-35,0$ & 10 \\
\hline$e_{1}^{5}$ & $40,10 n-41,1$ & 20 \\
\hline$e^{5}$ & $45,10 n-45,0$ & 10 \\
\hline$e_{1}^{6}$ & $50,10 n-50,0$ & 20 \\
\hline$e^{6}$ & $55,10 n-55,0$ & 10 \\
\hline$\vdots$ & $\vdots$ & $\vdots$ \\
\hline$e_{1}^{(n-2) / 2}$ & $5 n-20,5 n+20,0$ & 20 \\
\hline$e^{(n-2) / 2}$ & $5 n-15,5 n+15,0$ & 10 \\
\hline$e_{1}^{n / 2}$ & $5 n-10,5 n+10,0$ & 20 \\
\hline$e^{n / 2}$ & $5 n-5,5 n+5,0$ & 10 \\
\hline$\left.e_{1}^{(n+2}\right) / 2$ & $5 n, 5 n, 0$ & 10 \\
\hline
\end{tabular}

Table 2. The values of $n(u), n(v)$ and equidistant vertices, where $n \geq 8$. 
The exceptional cases are given in Table 3. Also, their Mostar indices are given in Table 4.

\begin{tabular}{cccccccccc}
\hline Type of edge & $\mathrm{C}_{40}$ & \multicolumn{3}{c}{$\mathrm{C}_{60}$} & \multicolumn{1}{c}{$\mathrm{C}_{80}$} \\
\hline$e_{1}^{1}$ & 12 & 12 & 16 & 12 & 12 & 36 & 12 & 12 & 56 \\
\hline$e^{1}$ & 9 & 20 & 11 & 9 & 39 & 12 & 9 & 59 & 12 \\
\hline$e_{1}^{2}$ & 15 & 22 & 3 & 18 & 40 & 2 & 18 & 60 & 2 \\
\hline$e^{2}$ & 15 & 25 & 0 & 15 & 45 & 0 & 15 & 65 & 0 \\
\hline$e_{1}^{3}$ & 18 & 18 & 4 & 23 & 34 & 3 & 24 & 54 & 2 \\
\hline$e^{3}$ & - & - & - & 25 & 35 & 0 & 25 & 55 & 0 \\
\hline$e_{1}^{4}$ & - & - & - & 29 & 29 & 2 & 32 & 47 & 1 \\
\hline$e^{4}$ & - & - & - & - & - & - & 35 & 45 & 0 \\
\hline$e_{1}^{5}$ & - & - & - & - & - & - & 39 & 39 & 2 \\
\hline
\end{tabular}

Table 3. Exception of $n(u), n(\mathrm{v})$, equidistant vertices.

\begin{tabular}{cccc}
\hline $\boldsymbol{n}$ & $\mathbf{4}$ & $\mathbf{6}$ & $\mathbf{8}$ \\
\hline $\mathrm{Mo}\left(\mathrm{C}_{10 n}\right)$ & 350 & 1360 & 3140 \\
\hline
\end{tabular}

Table 4. Special cases of Mostar index of fullerene $C_{10 n}$.

Now consider the fullerene graph $C_{10 n}$, where $n$ is odd, as depicted in Figure 2.2. There are five symmetry elements of order two in $\operatorname{Aut}\left(C_{10 n}\right)$ denoted by $\alpha_{i}, 1 \leq$ $i \leq 5$. One can easily check that

$f i x\left(\alpha_{1}\right)=\left\{1^{1}, 10^{2}, 10^{3}, \cdots, 10^{n}, 1^{n+1}, 5^{2}, 5^{3}, \cdots, 5^{n}\right\}$,

fix $\left(\alpha_{2}\right)=\left\{2^{1}, 2^{2}, 2^{3}, \cdots, 2^{n}, 2^{n+1}, 7^{2}, 7^{3}, \cdots, 7^{n}\right\}$,

fix $\left(\alpha_{3}\right)=\left\{3^{1}, 4^{2}, 4^{3}, \cdots, 4^{n}, 3^{n+1}, 9^{2}, 9^{3}, \cdots, 9^{n}\right\}$,

$f i x\left(\alpha_{4}\right)=\left\{4^{1}, 6^{2}, 6^{3}, \cdots, 6^{n}, 4^{n+1}, 1^{2}, 1^{3}, \cdots, 1^{n}\right\}$,

$f i x\left(\alpha_{5}\right)=\left\{5^{1}, 8^{2}, 8^{3}, \cdots, 8^{n}, 5^{n+1}, 3^{2}, 3^{3}, \cdots, 3^{n}, 2^{n+1}\right\}$.

This means that $f i x\left(\alpha_{i}\right)=2 n,(1 \leqslant i \leqslant 5)$. Similar to the last case, the presentations of other elements of $A u t\left(C_{10 n}\right)$ are as follows:

$\beta_{1}=\left(1^{1}, 1^{n+1}\right)\left(2^{1}, 2^{n+1}\right)\left(3^{1}, 3^{n+1}\right)\left(4^{1}, 4^{n+1}\right)\left(5^{1}, 5^{n+1}\right)\left(1^{2}, 1^{n}\right)\left(2^{2}, 2^{n}\right)\left(3^{2}, 3^{n}\right)\left(4^{2}, 4^{n}\right)$

$\left(5^{2}, 5^{n}\right)\left(6^{2}, 6^{n}\right)\left(7^{2}, 7^{n}\right)\left(8^{2}, 8^{n}\right)\left(9^{2}, 9^{n}\right)\left(10^{2}, 10^{n}\right)\left(1^{3}, 1^{n-1}\right)\left(2^{3}, 2^{n-1}\right)\left(3^{3}, 3^{n-1}\right)\left(4^{3}\right.$, $\left.4^{n-1}\right)\left(5^{3}, 5^{n-1}\right)\left(6^{3}, 6^{n-1}\right)\left(7^{3}, 7^{n-1}\right)\left(8^{3}, 8^{n-1}\right)\left(9^{3}, 9^{n-1}\right)\left(10^{3}, 10^{n-1}\right) \cdots\left(1^{(n+1) / 2}\right.$, $\left.1^{(n+3) / 2}\right)\left(2^{(n+1) / 2}, 2^{(n+3) / 2}\right)\left(3^{(n+1) / 2}, 3^{(n+3) / 2}\right)\left(4^{(n+1) / 2}, 4^{(n+3) / 2}\right)\left(5^{(n+1) / 2}, 5^{(n+3) / 2}\right)$ $\left(6^{(n+1) / 2}, 6^{(n+3) / 2}\right)\left(7^{(n+1) / 2}, 7^{(n+3) / 2}\right)\left(8^{(n+1) / 2}, 8^{(n+3) / 2}\right)\left(9^{(n+1) / 2}, 9^{(n+3) / 2}\right)$ $\left(10^{(n+1) / 2}, 10^{(n+3) / 2}\right)$,

$\beta_{2}=\left(1^{1}, 3^{n+1}\right)\left(2^{1}, 2^{n+1}\right)\left(3^{1}, 1^{n+1}\right)\left(4^{1}, 5^{n+1}\right)\left(5^{1}, 4^{n+1}\right)\left(1^{2}, 3^{n}\right)\left(2^{2}, 2^{n}\right)\left(3^{2}, 1^{n}\right)\left(4^{2}\right.$, $\left.10^{n}\right)\left(5^{2}, 9^{n}\right)\left(6^{2}, 8^{n}\right)\left(7^{2}, 7^{n}\right)\left(8^{2}, 6^{n}\right)\left(9^{2}, 5^{n}\right)\left(10^{2}, 4^{n}\right)\left(1^{3}, 3^{n-1}\right)\left(2^{3}, 2^{n-1}\right)\left(3^{3}, 1^{n-1}\right)$

$\left(4^{3}, 10^{n-1}\right)\left(5^{3}, 9^{n-1}\right)\left(6^{3}, 8^{n-1}\right)\left(7^{3}, 7^{n-1}\right)\left(8^{3}, 6^{n-1}\right)\left(9^{3}, 5^{n-1}\right)\left(10^{3}, 4^{n-1} \ldots\right.$ $\left(1^{(n+1) / 2}, 3^{(n+3) / 2}\right)\left(2^{(n+1) / 2}, 2^{(n+3) / 2}\right)\left(3^{(n+1) / 2}, 1^{(n+3) / 2}\right)\left(4^{(n+1) / 2}, 10^{(n+3) / 2}\right)$ 


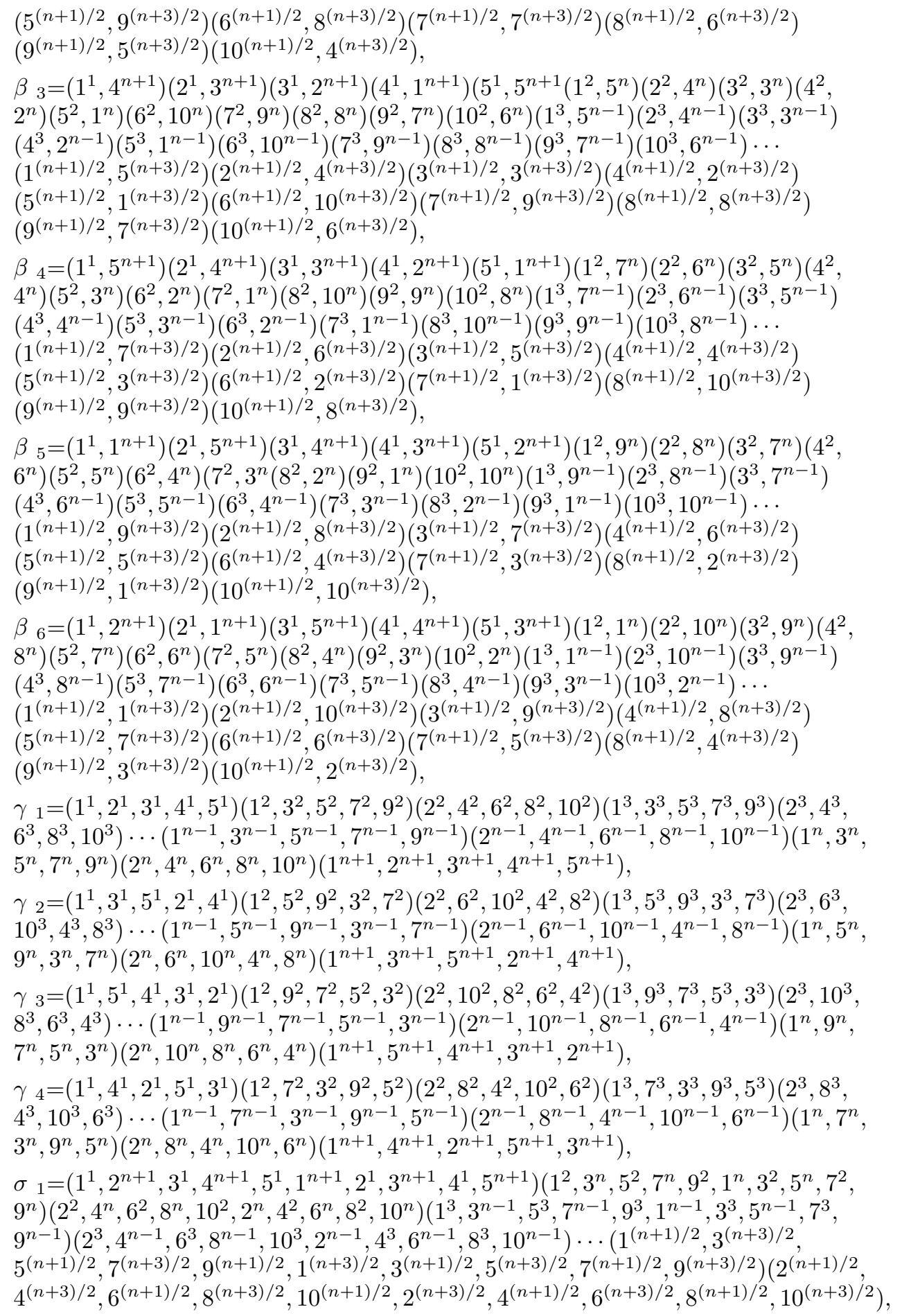


$\sigma_{2}=\left(1^{1}, 3^{n+1}, 5^{1}, 2^{n+1}, 4^{1}, 1^{n+1}, 3^{1}, 5^{n+1}, 2^{1}, 4^{n+1}\right)\left(1^{2}, 5^{n}, 9^{2}, 3^{n}, 7^{2}, 1^{n}, 5^{2}, 9^{n}, 3^{2}\right.$, $\left.7^{n}\right)\left(2^{2}, 6^{n}, 10^{2}, 4^{n}, 8^{2}, 2^{n}, 6^{2}, 10^{n}, 4^{2}, 8^{n}\right)\left(1^{3}, 5^{n-1}, 9^{3}, 3^{n-1}, 7^{3}, 1^{n-1}, 5^{3}, 9^{n-1}, 3^{3}\right.$, $\left.7^{n-1}\right)\left(2^{3}, 6^{n-1}, 10^{3}, 4^{n-1}, 8^{3}, 2^{n-1}, 6^{3}, 10^{n-1}, 4^{3}, 8^{n-1}\right) \cdots\left(1^{(n+1) / 2}, 5^{(n+3) / 2}\right.$, $\left.9^{(n+1) / 2}, 3^{(n+3) / 2}, 7^{(n+1) / 2}, 1^{(n+3) / 2}, 5^{(n+1) / 2}, 9^{(n+3) / 2}, 3^{(n+1) / 2}, 7^{(n+3) / 2}\right)\left(2^{(n+1) / 2}\right.$, $\left.6^{(n+3) / 2}, 10^{(n+1) / 2}, 4^{(n+3) / 2}, 8^{(n+1) / 2}, 2^{(n+3) / 2}, 6^{(n+1) / 2}, 10^{(n+3) / 2}, 4^{(n+1) / 2}, 8^{(n+3) / 2}\right)$, $\sigma_{3}=\left(1^{1}, 4^{n+1}, 2^{1}, 5^{n+1}, 3^{1}, 1^{n+1}, 4^{1}, 2^{n+1}, 5^{1}, 3^{n+1}\right)\left(1^{2}, 7^{n}, 3^{2}, 9^{n}, 5^{2}, 1^{n}, 7^{2}, 3^{n}, 9^{2}\right.$, $\left.5^{n}\right)\left(2^{2}, 8^{n}, 4^{2}, 10^{n}, 6^{2}, 2^{n}, 8^{2}, 4^{n}, 10^{2}, 6^{n}\right)\left(1^{3}, 7^{n-1}, 3^{3}, 9^{n-1}, 5^{3}, 1^{n-1}, 7^{3}, 3^{n-1}, 9^{3}\right.$, $\left.5^{n-1}\right)\left(2^{3}, 8^{n-1}, 4^{3}, 10^{n-1}, 6^{3}, 2^{n-1}, 8^{3}, 4^{n-1}, 10^{3}, 6^{n-1}\right) \cdots\left(1^{(n+1) / 2}, 7^{(n+3) / 2}\right.$, $\left.3^{(n+1) / 2}, 9^{(n+3) / 2}, 5^{(n+1) / 2}, 1^{(n+3) / 2}, 7^{(n+1) / 2}, 3^{(n+3) / 2}, 9^{(n+1) / 2}, 5^{(n+3) / 2}\right)\left(2^{(n+1) / 2}\right.$, $\left.8^{(n+3) / 2}, 4^{(n+1) / 2}, 10^{(n+3) / 2}, 6^{(n+1) / 2}, 2^{(n+3) / 2}, 8^{(n+1) / 2}, 4^{(n+3) / 2}, 10^{(n+1) / 2}, 6^{(n+3) / 2}\right)$,

$\sigma_{4}=\left(1^{1}, 5^{n+1}, 4^{1}, 3^{n+1}, 2^{1}, 1^{n+1}, 5^{1}, 4^{n+1}, 3^{1}, 2^{n+1}\right)\left(1^{2}, 9^{n}, 7^{2}, 5^{n}, 3^{2}, 1^{n}, 9^{2}, 7^{n}, 5^{2}\right.$, $\left.3^{n}\right)\left(2^{2}, 10^{n}, 8^{2}, 6^{n}, 4^{2}, 2^{n}, 10^{2}, 8^{n}, 6^{2}, 4^{n}\right)\left(1^{3}, 9^{n-1}, 7^{3}, 5^{n-1}, 3^{3}, 1^{n-1}, 9^{3}, 7^{n-1}, 5^{3}\right.$, $\left.3^{n-1}\right)\left(2^{3}, 10^{n-1}, 8^{3}, 6^{n-1}, 4^{3}, 2^{n-1}, 10^{3}, 8^{n-1}, 6^{3}, 4^{n-1}\right) \cdots\left(1^{(n+1) / 2}, 9^{(n+3) / 2}\right.$, $\left.7^{(n+1) / 2}, 5^{(n+3) / 2}, 3^{(n+1) / 2}, 1^{(n+3) / 2}, 9^{(n+1) / 2}, 7^{(n+3) / 2}, 5^{(n+1) / 2}, 3^{(n+3) / 2}\right)\left(2^{(n+1) / 2}\right.$, $\left.10^{(n+3) / 2}, 8^{(n+1) / 2}, 6^{(n+3) / 2}, 4^{(n+1) / 2}, 2^{(n+3) / 2}, 10^{(n+1) / 2}, 8^{(n+3) / 2}, 6^{(n+1) / 2}, 4^{(n+3) / 2}\right)$.

So, the fullerene $\mathrm{C}_{10 n}$ ( $n$ is odd) has $\frac{n-1}{2} \times 2+1=n$ orbits which each of them has 10 vertices, see Table 5 .

\begin{tabular}{cc}
\hline Vertex & Members of orbit \\
\hline $1^{1}$ & $1^{1}, 2^{1}, 3^{1}, 4^{1}, 5^{1}, 1^{n+1}, 2^{n+1}, 3^{n+1}, 4^{n+1}, 5^{n+1}$ \\
\hline $1^{2}$ & $1^{2}, 3^{2}, 5^{2}, 7^{2}, 9^{2}, 1^{n}, 3^{n}, 5^{n}, 7^{n}, 9^{n}$ \\
\hline $2^{2}$ & $2^{2}, 4^{2}, 6^{2}, 8^{2}, 10^{2}, 2^{n}, 4^{n}, 6^{n}, 8^{n}, 10^{n}$ \\
\hline$\vdots$ & $\vdots$ \\
\hline $1^{(n+1) / 2}$ & $1^{(n+1) / 2}, 3^{(n+1) / 2}, 5^{(n+1) / 2}, 7^{(n+1) / 2}, 9^{(n+1) / 2}$, \\
& $1^{(n+3) / 2}, 3^{(n+3) / 2}, 5^{(n+3) / 2}, 7^{(n+3) / 2}, 9^{(n+3) / 2}$ \\
\hline $2^{(n+1) / 2}$ & $2^{(n+1) / 2}, 4^{(n+1) / 2}, 6^{(n+1) / 2}, 8^{(n+1) / 2}, 10^{(n+1) / 2}$, \\
& $2^{(n+3) / 2}, 4^{(n+3) / 2}, 6^{(n+3) / 2}, 8^{(n+3) / 2}, 10^{(n+3) / 2}$ \\
\hline
\end{tabular}

Table 5. Members of orbits of $C_{10 n}, n$ is odd.

The values of $n(u), n(v)$ and equidistant vertices in Table 6 can be obtained by a similar argument. Hence, we have the following theorem.

Theorem 2.5. Consider the fullerene graph $C_{10 n}$ ( $n$ is odd), then there are $n+1$ orbits under the action of automorphism group on the set of edges. They are $O\left(e_{1}^{1}\right)$, $O\left(e^{1}\right), O\left(e_{1}^{2}\right), O\left(e^{2}\right), \cdots, O\left(e^{(n-1) / 2}\right), O\left(e_{1}^{(n+1) / 2}\right)$ and $O\left(e^{(n+1) / 2}\right)$.

Theorem 2.6. Consider the fullerene graph $C_{10 n}$ where $n$ is odd and $n \geq 9$. Then

$$
M o\left(C_{10 n}\right)=75 n^{2}-100 n+4005 .
$$




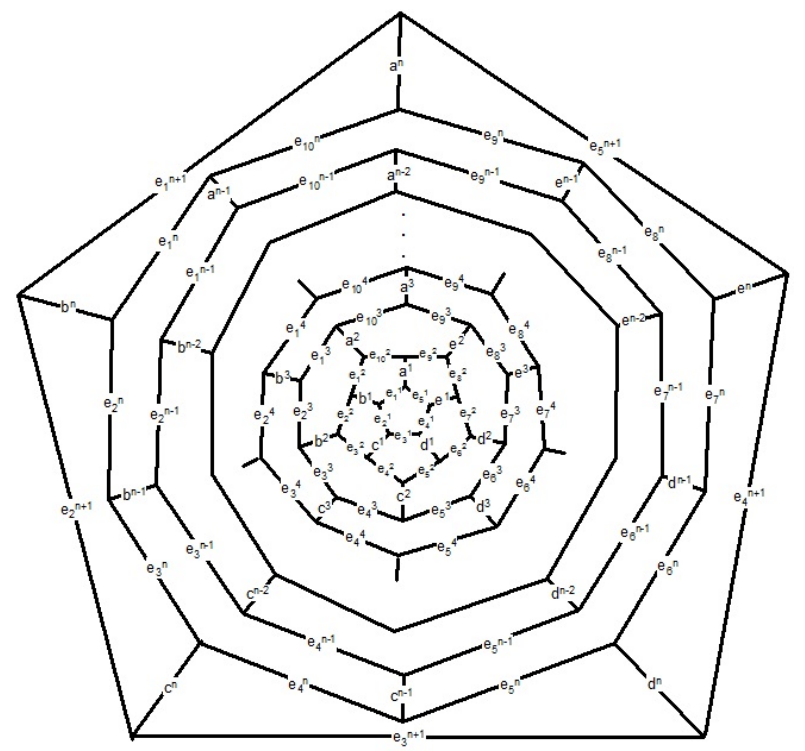

FIG. 2.4: $\mathrm{C}_{10 n}, n$ is odd.

\begin{tabular}{ccc}
\hline Type of edge & $\boldsymbol{n}(\boldsymbol{u}), \boldsymbol{n}(\boldsymbol{v})$, equidistant & Number \\
\hline$e_{1}^{1}$ & $12,12,10 n-24$ & 10 \\
\hline$e^{1}$ & $9,10 n-21,12$ & 10 \\
\hline$e_{1}^{2}$ & $18,10 n-20,2$ & 20 \\
\hline$e^{2}$ & $15,10 n-15,0$ & 10 \\
\hline$e_{1}^{3}$ & $24,20 n-26,2$ & 20 \\
\hline$e^{3}$ & $25,10 n-25,0$ & 10 \\
\hline$e_{1}^{4}$ & $32,10 n-33,1$ & 20 \\
\hline$e^{4}$ & $35,10 n-35,0$ & 10 \\
\hline$e_{1}^{5}$ & $40,10 n-41,1$ & 20 \\
\hline$e^{5}$ & $45,10 n-45,0$ & 20 \\
\hline$e_{1}^{6}$ & $50,10 n-50,0$ & 20 \\
\hline$e^{6}$ & $55,10 n-55,0$ & 10 \\
\hline$\vdots$ & $\vdots$ & $\vdots$ \\
\hline$e_{1}^{(n-1) / 2}$ & $5 n-15,5 n+15,0$ & 20 \\
\hline$e^{(n-1) / 2}$ & $5 n-10,5 n+10,0$ & 10 \\
\hline$e_{1}^{(n+1) / 2}$ & $5 n-5,5 n+5,0$ & 20 \\
\hline$e^{(n+1) / 2}$ & $5 n, 5 n, 0$ & 5 \\
\hline
\end{tabular}

Table 6. The values of $n(e), n(v)$ and equidistant vertices, where $n \geq 7$.

The exceptional cases are given in Table 7. Also, their Mostar indices are given in Table 8. 


\begin{tabular}{cccccccccc}
\hline Type of edge & $\mathrm{C}_{30}$ & \multicolumn{1}{c}{$\mathrm{C}_{50}$} & \multicolumn{7}{c}{$\mathrm{C}_{70}$} \\
\hline$e_{1}^{1}$ & 10 & 10 & 10 & 12 & 12 & 26 & 12 & 12 & 46 \\
\hline$e^{1}$ & 9 & 13 & 8 & 9 & 29 & 12 & 9 & 49 & 12 \\
\hline$e_{1}^{2}$ & 12 & 14 & 4 & 17 & 30 & 3 & 18 & 50 & 2 \\
\hline$e^{2}$ & 15 & 15 & 0 & 15 & 35 & 0 & 15 & 55 & 0 \\
\hline$e_{1}^{3}$ & - & - & - & 21 & 26 & 3 & 24 & 44 & 2 \\
\hline$e^{3}$ & - & - & - & 25 & 25 & 0 & 25 & 45 & 0 \\
\hline$e_{1}^{4}$ & - & - & - & - & - & - & 31 & 37 & 2 \\
\hline$e^{4}$ & - & - & - & - & - & - & 35 & 35 & 0 \\
\hline
\end{tabular}

Table 7. Exception of $n(u), n(\mathrm{v})$, equidistant vertices.

\begin{tabular}{cccc}
\hline $\boldsymbol{n}$ & $\mathbf{3}$ & $\mathbf{5}$ & $\mathbf{7}$ \\
\hline $\mathrm{Mo}\left(\mathrm{C}_{10 n}\right)$ & 80 & 760 & 2160
\end{tabular}

Table 8. Special cases of Mostar index of fullerene $C_{10 n}$.

\section{Acknowledgments}

The research of the first author is partially supported by the Shahid Rajaee Teacher Training University.

\section{REF E R E N C E S}

1. A. R. Ashrafi and M. Ghorbani: Computer application of GAP to the evaluation of numbers of permutational isomers of hetero fullerenes, MATCH Commun. Math. Comput. Chem. 60 (2) (2008) $359-367$.

2. A. R. Ashrafi and M. Ghorbani: Distance matrix and diameter of two infinite family of fullerenes, Optoelect. Adv. Mater-Rapid Commun. 3 (6) (2009) 596 - 599.

3. A. R. Ashrafi and M. Ghorbani: PI and omega polynomials of IPR fullerenes, Fuller. Nanotub. Carbon Nanostruct. 18 (3) (2010) 198 - 206.

4. A. R. Ashrafi, M. Ghorbani and M. Jalali: Eccentric connectivity polynomial of an infinite family of fullerenes, Optoelect. Adv. Mater-Rapid Commun. 3 (8) (2009) $823-826$

5. A. R. Ashrafi, M. Ghorbani and M. Jalali: Study of IPR fullerene by counting polynomials, J. Theo. Com. Chem. 8 (3) (2009) $451-457$.

6. A. R. Ashrafi, M. Ghorbani and M. Jalali: The PI and edge Szeged polynomials of an Infinite family of fullerenes, Fuller. Nanotub. Carbon Nanostruct. 18 (3) (2010) $107-116$.

7. A. R. Ashrafi, M. Ghorbani and M. Jalali: The vertex PI and Szeged indices of an Infinite family of fullerenes, J. Theor. Comput. Chem. 7 (2) (2008) $221-231$.

8. A. R. Ashrafi, M. Jalali, M. Ghorbani and M. V. Diudea:Computing PI and omega polynomials of an infinite family of fullerenes, MATCH Commun. Math. Comput. Chem. 60 (3) (2008) $905-916$. 
9. J. D. Dixon and B. Mortimer: Permutation groups, Graduate Texts in Mathematics, 163. Springer-Verlag, New York 1996.

10. T. Došlić, I. Martinjak, R. Škrekovski, S. T. Spužević and I. ZubaC: Mostar index, J. Math. Chem. 56 (2018) 2995 - 3013.

11. P. W. Fowler, D. E. Manolopoulos, D. B. Redmond and R. Ryan: Possible symmetries of fullerenes structures, Chem. Phys. Lett. 202 (1993) $371-378$.

12. M. Ghorbani and A. R. Ashrafi: Counting the number of hetero fullerenes, J. Comput. Theor. Nanosci. 3 (5) (2006) $803-810$.

13. M. Ghorbani, A. R. Ashrafi and M. Hemmasi: Eccentric connectivity polynomials of fullerenes, Optoelect. Adv. Mater-Rapid Commun. 3 (12) (2009) 1306 - 1308.

14. M. Ghorbani and M. Hemmasi: The vertex PI and Szeged polynomials of an infinite family of fullerenes, J. Comput. Theor. Nanosci. 7 (2010) $2405-2410$.

15. M. GHORBANI and M. JADDI: Computing counting polynomials of leapfrog fullerenes, Optoelect. Adv. Mater-Rapid Commun. 4 (4) (2010) 540 - 543.

16. M. Ghorbani and M. JALALI: Counting numbers of permutational isomers of an infinite family of fullerenes, Studia Ubb. Chemia 2 (2009) 145 - 152.

17. M. Ghorbani and M. Jalali: Counting nubers of permutational isomers of hetero fullerenes, Dig. J. Nanomat. Bios. 3 (4) (2008) $269-275$.

18. M. GHORBAni and M. SONGHORI: On the automorphism group of polyhedral graphs, Applied Math. Comput. 282 (2016) $237-243$.

19. M. Ghorbani and M. Songhori: Polyhedral graphs via their automorphism groups, Applied Math. Comput. 321 (2018) $1-10$.

20. M. Ghorbani, M. Songhori, A. R. Ashrafi and A. Graovać: Symmetry group of (3,6)-fullerenes, Fuller. Nanotub. Carbon Nanostruct. 23 (2015) $788-791$.

21. M. Jalali and M. Ghorbani: On omega polynomial of $C_{40 n+6}$ fullerenes, Studia Ubb. Chemia 4 (2009) $25-32$.

22. A. Khaksari, M. Hakimi-Nezhatd, O. Ori and M. Ghorbani: A survey of the automorphism groups of some fulleroids, Fuller. Nanotub. Carbon Nanostruct. 26 (2018) $80-86$.

23. H. W. Kroto, J. R. Heath, S. C. O’Brien, R. F. Curl and R. E. Smalley: $C_{60}$ : Buckminster fullerene, Nature 318 (1985) $162-163$.

\author{
Modjtaba Ghorbani \\ Department of Mathematics \\ Faculty of Science \\ Shahid Rajaee Teacher Training University \\ Tehran, 16785-136, I. R. Iran \\ mghorbani@sru.ac.ir \\ Shaghayegh Rahmani \\ Department of Mathematics \\ Faculty of Science \\ Shahid Rajaee Teacher Training University \\ Tehran, 16785-136, I. R. Iran \\ s.rahmani@sru.ac.ir
}

